\title{
Use of Reiki as a biofield therapy: An adjunct to conventional medical care
}

\author{
Sangeeta Singg* \\ Professor of Psychology, Angelo State University, San Angelo, USA
}

\begin{abstract}
Reiki is a noninvasive, nondiagnostic, and nondirective hands-on healing therapy which is believed to promote healing and a state of well-being in all living things. In recent years, its popularity has increased and as a biofield or energy therapy it is being used as a complementary modality with the conventional medical care in a variety of settings. Reiki is a Japanese term meaning universal life force which is believed to flow from the hands of a practitioner to a willing recipient's body facilitating physical, emotional, or spiritual healing depending on the needs of the recipient. This article provides a comprehensive review of the information available about Reiki in the areas of its history and development, nature of training and treatment protocol, practice ethics, empirical research and challenges for the researchers, guidelines for future research, and current applications. There is a vast amount of anecdotal, published, unpublished, and Internet information available on the subject of Reiki and its use as a healing modality with discrepancies among many sources. This article attempts to sort out and present only that information which is accepted by majority of the Reiki practitioners and as such is an informative reading for all health practitioners.
\end{abstract}

An increasing number of health care providers all over the world have begun integrating biofield (energy field) therapies such as Reiki, Healing Touch, Craniosacral, and Qigong into their patient care programs. The National Center for Complementary and Integrative Health (NCCIH), an US government health agency has classified Reiki as an energy medicine and a biofield therapy [1]. The use of biofield therapies in various forms have been a common practice in the ancient civilizations and Eastern societies. However, in 1960s, the modern Western world began embracing them as well. The notion is that biofield therapy facilitates health and well-being in living organisms by interacting with their biofield or energy field. Although different terms have been used to refer to the biofield therapies such as "alternative," "complementary," and "integrated" therapies, The NCCIH generally uses the term "complementary health approaches." A recent survey funded by the NCCIH reported that $55 \%$ of 31,000 participants believed that using a complementary therapy with the conventional medical treatments improved their overall health and $1.1 \%$ of them had used Reiki [2]. While the allopathic medicine is based on scientific inquiry, most of the alternative modalities such as Reiki are based on tradition and belief. In spite of the absence of a solid scientific basis, Reiki is being used as a complementary therapy in the treatment regimen of many patients worldwide. Unlike many other complementary and alternative therapies which require extensive training and supervision, Reiki requires very little training and practically no supervision after the training session, which makes it the second to prayer as the most accessible modality [3].

The word Reiki (pronounced Ray-key) is a generic Japanese term used in that culture to denote any healing system that involves energy work. More specifically, Rei means universal or omnipresent and $\mathrm{Ki}$ means life force or energy. This universal life force is believed to be all-knowing and all-encompassing which animates all living things. Other languages have similar words for this kind of universal life force, e.g., ch'i in Chinese, prana in Sanskrit, mana in Polynesian, pneuma in Greek, and ruah in Hebrew [3,4]. While the origins of Reiki practice are spiritual, it is not affiliated with any religion. The training and information about Reiki are easily available. There are several national and international Reiki organizations, many websites, and a large amount of published material. There is a professional association for the global Reiki community in existence for over 18 years named "The International Association of Reiki Professionals (IARP)". It has members from over 50 countries and provides extensive information about Reiki practice, code of ethics, liability insurance, research, and listing of practitioners. In spite of worldwide acceptance and practice, there is a lack of scientific evidence for the efficacy of Reiki therapy. Plus, most of the theoretical and historical information about Reiki lacks any written verification because Reiki practice is based on an oral tradition. The present author interviewed several practitioners and reviewed a large number of written sources to reconcile a vast amount of anecdotal information. The current article is an attempt to present a coherent and widely accepted version of Reiki origin, history, and practice. Also, a review of clinical trials to determine the current state of Reiki research and evidence for efficacy is presented.

\section{Conceptual framework}

There is an invisible field of energy surrounding all living things called biofield. Just as we do not see the energy field around a magnet, the biofield of living things is invisible to the human eye. The basic premise is that all living things are animated with the same universal life force, a vital energy of a spiritual source which is channeled through

Correspondence to: Sangeeta Singg, PhD, Professor of Psychology, Angelo State University, San Angelo, Texas 76909, USA, Tel: 1-325-223-8606; Fax: 1-325-9422290, E-mail: sangeeta.singg@angelo.edu

Key words: reiki, biofield therapy, alternative medicine, complementary therapy, eastern therapy, energy work, reiki healing, touch therapy

Received: March 10, 2015; Accepted: April 12, 2015; Published: April 14, 2015 
a Reiki practitioner's hands to promote energy balancing, healing, and a state of well-being. The Reiki practitioner remains neutral and makes no attempt to heal or change the biofield of the recipient. Anyone can learn Reiki, a professional or a layperson for self- and others' healing. Because the medical community is beginning to recognize the contribution of energy medicine in healing, Reiki is offered as a standard part of care in 76 US hospitals, many clinics, and hospice programs [5].

Being a noninvasive process, Reiki is believed to flow through a practitioner's hands to a willing recipient. The practitioner only serves as a channel for the flow of the universal life force without directing or controlling this energy. The transmission of this energy can be made by gentle physical touch or intention from any distance. Unlike those energy therapies that assess the recipient's biofield and make corrections, Reiki practitioners remain neutral without diagnosing or working on reorganizing the biofield. It is believed that Reiki is an intelligent life force which will reach the body part that needs it without any effort on the practitioner's part. The major difference between Reiki and other hands-on healing modalities is the nondirective stance of the Reiki practitioner [3]. Another difference between Reiki and other biofield therapies is the unique training procedure of the Reiki practitioner. Unlike other hands-on healing modalities, Reiki is not taught, instead, it is transferred from the Reiki master to the student during the induction process called attunement [6,7]. Attunement here means allowing oneself to open up to channel the universal life force. Once one receives the attunement to channel Reiki, it is believed that one never loses it. However, some believe that you do not need to be attuned to channel Reiki because the ability to tap into the universal life force is inborn in all of us. There are many ways to tap into this universal life force and Reiki appears to be one of the easiest and quickest ways to achieve this [3].

In order for Reiki to be of any benefit, a recipient has to be willing to receive it and it is unethical to send Reiki to an unwilling recipient. However, if the recipients are children, animals, or seriously ill persons who cannot express their will in this regard, then it is acceptable to transmit Reiki to them. They will let the practitioner know by moving away from the practitioner's hands or by not accepting it if they do not want it [4].

Reiki is experienced differently by different recipients. Most commonly reported sensations are warmth, tingling, or coolness followed by a general relaxation. Some feel desired change in symptoms after one session and others take a longer period of time with multiple sessions. No negative side effects are reported with Reiki treatment. The assumption is that with a willing recipient, Reiki will work for the recipient's highest good because the intelligent life force knows what is best for the person [4,7]. Reiki is believed to work simultaneously at the physical, mental, and spiritual levels. Also, transmission of Reiki benefits the practitioner when the universal life force is channeled through his or her hands. Thus, it benefits both the practitioner and the recipient.

\section{Origin and history of Reiki}

A Japanese spiritualist named Mikao Usui (1865-1926), the founder of the Usui System of Reiki, was prompted to begin a quest for information on healing. He studied Tibetan Buddhist Sanskrit writings in the form of Sutras, which explained how the healing was done some 2500 years ago. Although he had found the information about the mechanisms of healing, he did not feel empowered to heal others. He then adopted another ancient Indian method of achieving enlightenment by performing Tapasya (penance by fasting and meditating) on Mount Koriyama, near Kyoto. After 21 days of Tapasya, Usui was enlightened with the knowledge of Reiki healing. Many people benefited from his knowledge of healing in Japan. He gave five spiritual precepts to his students for their daily life: do not anger, do not worry, be humble, be honest in your work, and be compassionate to yourself and others [3,4,6-8].

Usui trained over 2000 students (18-19 masters) including Dr. Chujiro Hayashi (1878-1940), a retired naval officer who founded a Reiki clinic in Tokyo in 1922 and trained 17 masters. Hawayo Takata (1900-1980), a Japanese-American was treated in Hayashi's clinic for relief from an illness while she was visiting her family in Japan. After her recovery, she received Reiki training by Hayashi and returned to Hawaii in 1937. Hayashi followed her to Hawaii and under his guidance in February 1938, she was named a Reiki master. In 1940 just before his death, Hayashi named Takata as his successor [3]. Takata practiced Reiki in Hawaii and later moved to California where she trained 22 Reiki masters including her granddaughter, Phyllis Furomoto. After her death, two leading Reiki masters created two different branches of the Usui system of Reiki. Furomoto founded The Reiki Alliance in 1981 and another student of Takata, Dr. Barbara Weber Ray founded the American International Reiki Association, Inc in 1982 [3,4,6,8]. At the present time, traditional Usui Reiki and several Western versions can be located on the Internet. Even though the Reiki practice has become more structured and formalized now than the original oral intuitive method, the goal still remains the personal and global healing as set by Usui. Takata maintained the oral tradition and did not allow her students to take any notes during the training sessions. This changed after her death. Different Reiki masters began creating their own manuals in an attempt to personalize their training for their students. Several claim to have more refined Reiki systems branded with their unique names $[3,4,6,8,9]$. Usui, Hayashi, and Takata are considered the three Grand Masters and because Takata did not appoint a successor, some have tried to claim the title of grand master [3,4]. There are several directories available on the Internet listing Reiki practitioners and masters. For example, The Reiki Page [10] lists its registered practitioners and masters from all over the world.

\section{Reiki attunement}

Traditional Reiki training was divided into three levels, Shoden (first teachings), Okuden (inner teachings), and Shinpiden (mystery teachings) which are now called first, second, and third (Reiki master) degrees. Some proponents of Western Reiki variations have changed the classification and training system according to their preferences. Currently, Reiki training is provided at three to four levels presented in 3 to 10 stages depending on a Reiki master [3,4]. In spite of the variations in the training methods, at every level a series of attunements are given which prepare a student's body for channeling the universal life force. Attunement process is believed to open the crown, heart, and palm chakras and create a special connection between the student and the Reiki source to channel Reiki from the top of one's head through the palms of the hands $[3,5]$. There are no formal examinations for the degrees because the main part of the training is the experience of attunement. Students receive a manual at each level which provides specific information pertaining to the level, e. g., the first degree manual may contain different hand positions along with the history, evidence for efficacy, code of ethics, etc. The number and sequence of laying hands on the body may differ from master to master ranging from 5 to $15[3,4,6,8]$. 
In the first degree, hand positions are shown and a series of attunement are given. In the second degree, additional attunements are given and a series of special symbols are revealed that allows for an ability to send Reiki to a distant source. The third degree training is for the experienced Reiki practitioners to learn a final symbol for attuning others which empowers them to become Reiki masters who can provide all three levels of Reiki training to others $[3,4,6,8]$. Each Reiki training workshop may last from a half day to several days. Certificates of completion are provided at the end of each workshop for each level of Reiki training.

\section{Reiki treatment}

Reiki is administered with the palms slightly cupped and placed directly or floated above the body of a fully clothed recipient. The hands are placed on a recipient's body in a sequential pattern covering the head, front, back, and feet. This way the Reiki is provided to the body's major organs, endocrine system, and lymphatic system. A Reiki session may last from 60 to 90 minutes [3,4,6,7]. Depending on the situation, sometimes the Reiki touch may last for only a few moments. This is very beneficial to those professionals such as nurses who serve a large number of patients and can only have a limited time with each patient. Reiki training is accepted as part of the continuing education for nurses in the United States [11]. When a recipient may not wish to be touched or is burned, Reiki can be administered by holding hands 3 to 4 inches over the recipient's body $[3,4,6]$.

\section{Scientific evidence of efficacy}

Currently there is a paucity of controlled research on efficacy of Reiki. In 1997, the author obtained training at all levels, from first degree to master level, and conducted and published a groundbreaking Reiki study with her graduate student Linda Dressen [6,8]. The author first learned about Reiki at the 1997 Annual Conference of the International Society for the Study of Subtle Energies and Energy Medicine (ISSSEEM). Because the author is originally from India, she was exposed to similar healing practices that have been in existence for centuries in India that do not have evidence-based practice except anecdotal reports. However, with research-based training and three graduate degrees in the United States, she considers herself a scientistpractitioner (licensed psychologist in the State of Texas) adhering to only what is supported by research. But her exposure to Reiki at the ISSSEEM revived the interest in energy work. The attendees at the ISSSEEM were medical doctors, nurses, psychologists, bioengineers, professional counselors, social workers, and laypersons who practiced Reiki and other complementary and alternative therapies. They inspired the author with their testimonies and she received her first degree in Reiki on July 20, 1997 in Dallas, Texas. She could not wait to share her experiences with her students about the new learned art of healing called Reiki. She also shared her frustration about the lack of scientific evidence for efficacy of Reiki practice and desire to conduct research in this area. A graduate student Linda Dressen who happened to be a Reiki master got inspired and enrolled in an independent research class with the author and participated in the Reiki research project. The author designed and directed the study in which five Reiki masters and four laypersons helped with collecting data from chronically ill patients in the West Texas area. The author prepared the report and presented the results at the Ninth Annual Conference of the ISSSEEM in Boulder, CO. From over 110 submissions, our paper [9] was one of the four technical papers accepted for the 1999 ISSSEEM conference. Our study was also published in Subtle Energies \& Energy Medicine [6]. A summary of the study is presented below.
This was the first experimental study that compared Reiki with progressive muscle relaxation, control, and placebo (sham Reiki) conditions and examined the efficacy of Reiki for emotional, personality, and spiritual changes in chronically ill patients [3]. Two independent variables were Sex and type of treatment and treatment groups were Reiki group (R), progressive muscle relaxation group (PMR), wait-list control group $(\mathrm{C})$, and placebo group $(\mathrm{P})$. The dependent variables included present pain intensity; total pain rating index-R (PRI-R); PRI-R: sensory quality of pain; PRI-R: affective quality of pain; PRI-R: evaluative quality of pain, depression, state anxiety, trait anxiety, selfesteem, locus of control, realistic sense of personal control, and belief in God's (higher power) assistance. The following instruments were used to measure these variables: General Information Questionnaire (GIQ), Social Readjustment Rating Scale, McGill Pain Questionnaire, Beck Depression II Inventory, State-Trait Anxiety Inventory, Rotter I-E Scale, Rosenberg Self-Esteem Scale, and Belief in Personal Control Scale [6].

The sample consisted of 48 men and 72 women $(N=120)$ who were chronically ill with headaches $(45 \%)$, heart disease (10\%), cancer (8\%), arthritis (7\%), peptic ulcer (6\%), asthma (7\%), hypertension (12\%), or human immunodeficiency virus (HIV) infection (5\%). They were predominantly Caucasian (92\%) with average age of 41.34 years $(S D=11.32)$. These participants had no prior experience with Reiki, PMR, or any type of hands-on healing therapy, and they all experienced some type of pain. These volunteers were randomly assigned to one of the four treatment groups resulting in eight (treatment $\mathrm{x}$ sex) subgroups $(n s=30)$. All participants received 10 30-minute sessions twice a week. The $\mathrm{R}$ group received Reiki sessions and the PMR group received sessions of PMR and deep breathing exercises. The participants in group $C$ read any material of their choice for 1030 -minute sessions and did not receive Reiki treatment. The group P received false (sham) Reiki treatments by four lay assistants who did not receive Reiki attunement, but learned the hand positions used on group R participants. The group $\mathrm{R}$ participants were contacted after 3 months for follow-up testing to assess the change in dependent measures from post-test to followup [6].

The $4 \times 2$ factorial ANOVA and Omega Squared $\left(\omega^{2}\right)$ for pretest/ post-test change for all dependent variables revealed significant results for 10 independent variables. Omega Squared, a measure of effect size provides the proportion of variance explained by the treatment variable. Only three main effects showed medium and four main effects showed large effect sizes. Large treatment effects (Omega Squared of .15 or larger is considered large treatment) were found on present pain intensity (.18), depression (.34), and state (.28) and trait (.29) anxiety. Medium treatment effects were noted on PRI-R: evaluative (.13), locus of control (.10), and unrealistic sense of control (.07); while treatment effects were small on PRI-R: sensory (.05), self-esteem (.05), and faith in God (.02). Significant interaction effects of treatment $\mathrm{x}$ sex were found only on depression and faith in God. Tukey/Kramer procedure was used for all post hoc pairwise comparisons. The post-test and follow-up comparison results of group $\mathrm{R}$ showed significant reduction in sensory and affective qualities of pain along with the overall pain measure. All other comparisons did not yield significant results.

This was the first study of Reiki that randomly assigned men and women experiencing similar levels of life-event stress to experimental conditions and compared Reiki with PMR therapy, no therapy, and false Reiki. One of the major contributions of this study was to demonstrate how a placebo group can be used in Reiki studies. This contribution was also recognized by the Touchstone Process team to be 
discussed later $[1,3,5]$. No study prior to this study had used a placebo group (false Reiki). Several studies designed experiments and quasiexperiments including a placebo or "sham Reiki" group after our study.

The limitations of the study included using a self-selected sample, multiple experimenters, multiple sites, and uncontrolled variables of religiosity and social support available to the patients. However, the random assignment of the participants and other controls used in the study provided some safeguards $[3,6]$. Six major conclusions of the study are presented below.

1. Reiki is an effective modality for the reduction of pain, depression, and state anxiety.

Men have greater reduction in depression than women after receiving Reiki.

2. Reiki facilitates desirable changes in personality. Recipients report decreased trait anxiety, self-esteem enhancement, and greater sense of internal locus of control. Further, their belief in their personal control becomes more realistic.

3. Reiki enhances recipient's faith that God is a powerful agent whose help can be enlisted. Women experience this enhancement in faith more than men.

4. Attunement is necessary for practice of Reiki. A sham Reiki practice would not be effective in facilitating desirable changes in pain, affective states, personality traits, and spirituality.

5. The gains made by Reiki persist over longer periods of time. Reduction in sensory and affective qualities of pain and the Total Pain Rating Index tend to persist even at 3-Month interval.

6. Chronically ill patients experiencing high stress and pain would be receptive to Reiki therapy $[3,6]$.

After our groundbreaking research, only a handful of scientific studies emerged. The studies examined heterogeneous physical and emotional problems using heterogeneous research instruments which made it difficult to compare results. Also, due to the challenges encountered by the researchers in this area, most of the studies have small samples and methodological limitations $[3,6]$. Five articles published between 2007 and 2014 conducted systematic reviews of clinical trials using Reiki therapy [1,3,12-15]. They used online search, specific criteria for inclusion, and comparative review of the studies. However, in her review of these reviews, the author noted that two articles eliminated animal studies and focused either only on randomized clinical trials [13] or on studies with test/control groups [14]. which in her opinion left out some important information presented in the eliminated studies. The three reviews discussed below present a well organized and comprehensive knowledge of Reiki research.

In 2008, The Center for Reiki Research (CRS) recruited a team of 7 researchers with doctoral degrees and 5 experienced nurses for "The Touchstone Process" to conduct a rigorous

review of all studies published up to June 2009 in the peer reviewed journals in the United States [1]. The Touchstone Process includes experts who conduct an ongoing critique of all Reiki research published in peer-reviewed journals. If a study met the selection criteria set by the team, it was reviewed by two independent doctoral level researchers before being included in a final group of evidence-based studies. The team identified a total of 26 peer-reviewed Reiki articles ( 7 qualitative and 19 quantitative). A summary of the Touchstone Process evaluation and article summaries are posted on the CRS website [5]. The author considers this the most comprehensive and systematic review in the area of Reiki research. Of the 26 studies that entered the Touchstone Process, only 12 articles [6,16-26] were assessed to have "robust research designs and well-established outcome parameters" [1]. These articles were assessed as "Very Good" or "Excellent" by at least 1 reviewer and were not considered "weak" by any reviewer of the Touchstone Process team $[3,5]$. Our study summarized above was included in their list of 12 finalists. The Touchstone Process team concluded that $83 \%$ of these "top range" studies showed "moderate to strong evidence in support of Reiki therapy for conditions relating to pain, stress, anxiety and mood [5].

The CRS Touchstone Process team also concluded that two carefully controlled studies on rats $[16,20]$. yielded the "strongest demonstrable biological effect." The results of these studies supported the assumption that Reiki significantly reduces stress as compared to sham Reiki. The team recognized the advantage of animal studies over the human studies because most intervening variables can be controlled in the laboratory setting and the results can eliminate the placebo effect $[1,5]$. In an effort to control some intervening variables, Baldwin and Schwartz [16] in their first experiment divided 16 male Sprague Dawley rats into four groups kept in four separate rooms: No Noise group, Noise group, Noise and Sham Reiki group, and Noise and Reiki group. Three of the four groups were subjected to 15 minutes of 90 $\mathrm{dB}$ white noise for 3 weeks and the fourth group was the quiet control group. Loud noise stress can damage the mesenteric microvasculature causing leakage of plasma into the surrounding tissue and the experiment was designed to determine whether Reiki as a healing energy can reduce the microvascular leakage. The Noise and Reiki group received a daily 15-minute Reiki treatment prior to the noise. In this experiment, Reiki significantly reduced the average number and area of microvascular leaks compared to the other noise groups. Baldwin et al. [20] conducted the second experiment by using three male Sprague-Dawley rats implanted with radiotelemetric transducers to monitor the heart rate and blood pressure. The rats were subjected to $90 \mathrm{~dB}$ white noise for 30 minutes a day for 8 days and during the last 5 days, two Rieki practitioners provided 15 minutes of Reiki before and 15 minutes after the noise. The experiment was repeated with the sham Reiki on the same animals. Mean heart rate and blood pressure were recorded before, during and after Reiki treatment and during the noise. Compared to the baseline, heart rate was significantly reduced after receiving Reiki than receiving sham Reiki, but there was no significant effect on the blood pressure. The authors concluded that Reiki was effective in modulating heart rate in rats which coincides with Reiki's effect as a stress reducer in humans.

Another major review was conducted by Thrane and Cohen [15] who examined the effect of Reiki for pain and anxiety in randomized clinical trials. Studies published in 2000 or later in peer-reviewed journals that used randomization and a control group to examine the effect of Reiki on pain or anxiety were included. Of the 12 articles on the subject, only 7 met the selection criteria (4 with cancer patients, 1 with post-surgical patients, and 2 with older adults). Most of the 7 studies yielded statistically significant results either for pain or anxiety or both. Cohen's d was used to calculate effect sizes and the effect sizes ranged from $\mathrm{d}=0.24$ (small) to $\mathrm{d}=2.08$ (large). The authors concluded that there is evidence to support the claim that Reiki therapy may be effective for pain and anxiety.

An integrative review of 16 Reiki studies published from 1980 
to 2006 was undertaken by Vitale [12]. Of these studies, 7 showed efficaciousness of Reiki therapy for stress reduction and depression [27], anxiety reduction $[6,26,28]$ and pain reduction $[6,25,29,30]$. Upon completion of her review, Vitale questioned the appropriateness of the randomized controlled trial design for examining the efficacy of energy work. Gaus and Hogel [31] identified some common challenges for designing studies of conventional therapies such as unfeasibility of placebo; unreliability of outcome variables; individual nature of the therapeutic applications; and problems with masking the therapy, obtaining large samples and providing long term treatments. NieldAnderson and Ameling [32] stated that these issues also apply to Reiki research and they identified some additional challenges. Their contention is that randomization and group assignments violate the individualized nature of the treatment plan which may be viewed as a threat to the practitioner-client relationship. Another challenge is that most Reiki practitioners do not have traditional research training or links with institutions conducting the research and are often left out of the loop. Unavailability of a standardized treatment protocol is another problem for replicable studies with Reiki therapy. Thus, there are some valid methodological and philosophical reasons that explain why it is difficult to conduct research on the efficacy of Reiki therapy [32].

Furthermore, some contend that it is difficult to demonstrate whether sham Reiki used as a placebo is truly inert or it introduces another confounding variable $[30,33]$ because human touch is healing in itself. The clinical trials focus on health conditions and symptoms, but Reiki therapy, holistic in nature moves toward balancing the overall system and empowering the body for self-healing. The phenomenological findings of some studies suggest that using qualitative research methods may be better approach to understanding the effects of Reiki [30]. Some other researchers have questioned the use of linear research methods to study biofield therapies [33-35]. It is suggested that an experimental design may not be optimal for studying an individualized healing therapy such as Reiki which is best used in a natural setting [27]. Perhaps all these researchers have valid concerns.

The Touchstone Process team [1] identified the small sample size to be the major weakness in all studies they evaluated. This problem exists because the studies are exploratory in nature and lack funding. Research guidelines including Essential Components of Good Study Design for future Reiki research by The Touchstone Process team are posted on the CRS website. The team is also available for consultation to those who may be seeking help in designing their studies [5].

The following conclusions of the Touchstone Process team offer acceptable answers to what we have learned from the Reiki research to date.

1. The measures used in research to date may not be sensitive enough for capturing individualized experiences of Reiki recipients because of the subtle and complex nature of Reiki practice.

2. There are no reported negative side effects of Reiki.

3. There is enough scientific evidence showing that Reiki is effective in reducing anxiety and pain, inducing relaxation, improving fatigue and depressive symptoms, and enhancing overall wellbeing [5].

\section{Current state of Reiki practice and applications}

Reiki therapy is being used in many hospitals and medical clinics worldwide and achieving recognition by medical doctors and nurses who are using it as an adjunct to conventional medical care in a variety of settings. Hospitals are using Reiki for patient relaxation and to decrease recovery time from injury, illness or surgery. Also, it is being used to reduce stress and manage pain for the chronically ill patients $[4,7,36]$. In many hospices for facilitation of the dying process and in many hospitals with cancer patients, Reiki promotes emotional healing and attitudinal changes $[4,7,36,37]$. Some are using it as a therapeutic tool for both the therapist and the client, and for therapeutic interaction in psychotherapy [35,38]. According to Barnett and Chambers [7], the Reiki therapy produces a sense of well-being and empowerment in the client and aids to release emotional residue from his or her body. Because of these medical and psychological applications, Reiki training is being accepted as continuing education for many types of helping professionals such as nurses and psychologists.

In the last few years, many hospitals have incorporated Reiki as an adjunct therapy for patient care with benefits such as pain reduction, anxiety reduction, faster recovery, lessened blood loss during surgery, fewer side effects of chemotherapy, and less use of medications. In mid 1990s, Reiki was used in the hospital operating rooms and is now offered as a regular part of patient services in $15 \%$ of the hospitals in the United States [39]. A detailed description of 64 Reiki hospital programs is posted on the CRS website [5].

The International Association of Reiki Professionals (IARP) has attempted to regulate the practice of Reiki by creating a membership registration, a code of ethics, and a liability insurance program [40]. The liability insurance program covers Reiki practitioners as well as teachers in the United States and Canada. In addition to providing the protection and support for IARP members, the insurance program makes them employable by the hospitals and other health care settings which require Reiki practitioners to have liability insurance to practice.

\section{Guidelines for future research}

Although there is a general agreement that it is difficult to conduct controlled scientific research on the efficacy of Reiki, but the several researchers discussed earlier have found the ways to randomly assign participants and use placebo and control groups. An issue pointed by Nield-Anderson and Ameling [32] of randomization and group assignment rendering participants without choice in treatment can be dealt with a crossover experimental design and a wait-list control group as was done by several studies [e.g., 6,13-15]. Nield-Anderson and Ameling also stated that the studies of efficacy of Reiki "deal almost exclusively with well populations.” This is not completely true. Several studies have used the patient samples [e.g., 6,15-30].

Notwithstanding, in spite of several clinical trials, there are still many research questions unanswered regarding the process and outcome of Reiki therapy. Some questions that may be considered are presented below.

(1) Since the assumption is that Reiki is a self-directing and intelligent force that goes where it is needed, is single hand position as opposed to multiple hand positions sufficient?

(2) Does the practitioner need to be focused and paying attention while going through the hand positions? The assumption is that no attention is required by the practitioner during the Reiki session because all one needs is to place the hands on another, and Reiki will go where it is needed.

(3) What is the optimal length of time for a Reiki session?

(4) What is the optimal length of time to hold a hand position? 
(5) Are there certain hand positions better for certain disorders?

(6) Is lying down a better position for receiving Reiki than a sitting or standing position?

(7) Are attunements reversible if a person wishes to have them removed $[3,4,6,8]$.

\section{Conclusion}

Reiki is a noninvasive, nondiagnostic, and nondirective hands-on healing therapy which is believed to promote healing and a state of well-being in all living things. Its popularity has increased in recent years because it is a simple modality that can be learned by anyone. Although the origin of Reiki is spiritual, it does not adhere to any particular religious tenets and is applicable at all wellness levels and on all living organisms. The first degree provides all that one needs to facilitate healing in oneself or others. However, second and third degrees can be valuable to those who desire to experience Reiki at deeper levels and teach others. There is enough research evidence to support that Reiki facilitates relaxation, reduces pain and anxiety, promotes healing, improves fatigue and depression, and enhances overall wellbeing. Although there has been an increased interest among researchers to examine the efficacy of Reiki therapy, more well-designed and controlled studies are needed for it to be accepted within the mainstream health care system. Because of the simplicity of this modality, the author will not be surprised if in the near future, the mainstream health care professionals begin advising the use of Reiki along with a balanced diet and exercise for prevention and treatment of illnesses. However, over commercialization of Reiki and ridiculing it by opening websites where people can learn it in 15 minutes attract some valid criticism of this modality. Benor [41] listed three limitations of Reiki therapy: inadequate preparation of later generations of masters, lack of formal structure for supervision/certification, and high fees for master level training. Greed may be the cause of several of these issues with the current state of Reiki therapy. Therefore, it is the responsibility of all Reiki practitioners to preserve the integrity of their practice, maintain high ethical standards, and refrain from competitive and gimmicky commercialization [3]. Conducting research using larger sample sizes, randomized groups, and standardized treatment protocols will also enhance the status of Reiki therapy.

\section{References}

1. Baldwin AL, Vitale A, Brownell E, Scicinski J, Kearns M, et al. (2010) The Touchstone Process: an ongoing critical evaluation of reiki in the scientific literature. Holist Nurs Pract 24: 260-276. [Crossref]

2. National Center of Complementary and Integrative Health (Accessed March 10, 2015) Available at http://www. nccih.nih.gov/health.

3. Singg S (2008) Reiki: An alternative therapy. In: Carol MD (Ed.), Complementary Therapies in Rehabilitation: Evidence for Efficacy in Therapy, Prevention, and Wellness (3rdedn), Thorofare, NJ: SIACK Inc.

4. Rand WL (2000) Reiki, the healing touch: first and second degree manual (3rdedn), Southfield, MI: Vision Publications.

5. The Center for Reiki Research: Including Reiki in Hospitals. Available at http://www.centerforreikiresearch.org.

6. Dressen LJ, Singg S (1998) Effects of Reiki on pain and selected affective and personality variables of chronically ill patients. Subtle Energies \& Energy Medicine 9: 51-82.

7. Barnett L, Chambers M (1996) Reiki Energy Medicine: Bringing Healing Touch into Home, Hospital, and Hospice. Rochester, VT: Healing Arts Press.

8. Haberly HJ (1990) Reiki: HawayoTakata's Story. Garrett Park, MD: Archedigm Publications.
9. Singg S, Dressen LJ (1999) Desirable self-perceived psychophysiological changes in chronically ill patients: an experimental study of Reiki. Presented at: Ninth Annual Conference of the International Society for the Study of Subtle Energies and Energy Medicine, Boulder, Colo.

10. The Reiki Page. Available at www.thereikipage.com/directory/index.php.

11. van Sell SL (1996) Reiki: an ancient touch therapy. $R N$ 59: 57-59. [Crossref]

12. Vitale A (2007) An integrative review of Reiki touch therapy research. Holist Nurs Pract 21: 167-179. [Crossref]

13. Lee MS, Pittler MH, Ernst E (2008) Effects of reiki in clinical practice: a systematic review of randomised clinical trials. Int J Clin Pract 62: 947-954. [Crossref]

14. Vander Vaart S, Gijsen VM, de Wildt SN, Koren G (2009) A systematic review of the therapeutic effects of Reiki. J Altern Complement Med 15: 1157-1169. [Crossref]

15. Thrane S, Cohen SM2 (2014) Effect of Reiki therapy on pain and anxiety in adults: an in-depth literature review of randomized trials with effect size calculations. Pain Manag Nurs 15: 897-908. [Crossref]

16. Baldwin AL, Schwartz GE (2006) Personal interaction with a Reiki practitioner decreases noise-induced microvascular damage in an animal model. J Altern Complement Med 12: 15-22. [Crossref]

17. Vitale A (2009) Nurses' lived experience of Reiki for self-care. Holist Nurs Pract 23 129-141, 142-1455. [Crossref]

18. Whelan KM, Wishnia GS (2003) Reiki therapy: the benefits to a nurse/Reiki practitioner. Holist Nurs Pract 17: 209-217. [Crossref]

19. Assefi N, Bogart A, Goldberg J, Buchwald D (2008) Reiki for the treatment of fibromyalgia: a randomized controlled trial. J Altern Complement Med 14: 1115-1122. [Crossref]

20. Baldwin AL, Wagers C, Schwartz GE (2008) Reiki improves heart rate homeostasis in laboratory rats. J Altern Complement Med 14: 417-422. [Crossref]

21. Vitale AT, O'Connor PC (2006) The effect of Reiki on pain and anxiety in women with abdominal hysterectomies: a quasi-experimental pilot study. Holist Nurs Pract 20: 263-272. [Crossref]

22. Crawford SE, Leaver VW, Mahoney SD (2006) Using Reiki to decrease memory and behavior problems in mild cognitive impairment and mild Alzheimer's disease. J Altern Complement Med 12: 911-913. [Crossref]

23. Shiflett SC, Nayak S, Bid C, Miles P, Agostinelli S (2002) Effect of Reiki treatments on functional recovery in patients in poststroke rehabilitation: a pilot study. J Altern Complement Med 8: 755-763. [Crossref]

24. Shore AG (2004) Long-term effects of energetic healing on symptoms of psychological depression and self-perceived stress. Altern Ther Health Med 10: 42-48. [Crossref]

25. Wirth DP, Brenlan DR, Levine RJ, Rodriguez CM (1993) The effect of complementary healing therapy on postoperative pain after surgical removal of impacted third molar teeth. Complement Ther Med 1: 133-138.

26. Witte D, Dundes L (2001) Harnessing life energy or wishful thinking? Reiki, placebo Reiki, meditation, and music. Altern Complement Ther 7: 304-309.

27. Wardell DW, Engebretson J (2001) Biological correlates of Reiki Touch(sm) healing. J Adv Nurs 33: 439-445. [Crossref]

28. Thornton L (1996) A study of Reiki, an energy field treatment, using Roger's science. Rogerian Nurs Sci News 8: 14-15.

29. Brewitt B, Vittetoe T, Hartwell B (1997) The efficacy of Reiki hands-on healing improvements in spleen and nervous system function as qualified by electrodermal screening. 3: 89 .

30. Olson K, Hanson J, Michaud M (2003) A phase II trial of Reiki for the management of pain in advanced cancer patients. J Pain Symptom Manage 26: 990-997. [Crossref]

31. Gaus W, Högel J (1995) Studies on the efficacy of unconventional therapies. Problems and designs. Arzneimittelforschung 45: 88-92. [Crossref]

32. Nield-Anderson L, Ameling A (2000) The empowering nature of Reiki as a complementary therapy. Holist Nurs Pract 14: 21-29. [Crossref]

33. SC, Nayak S, Bid C, Miles P, Agostinelli S (2002) Effect of Reiki treatments on functional recovery in patients in poststroke rehabilitation: a pilot study. J Altern Complement Med 8: 755-763. [Crossref] 
34. Schiller R (2003) Reiki: a starting point for integrative medicine. Altern Ther Health Med 9: 20-21. [Crossref]

35. Miles P, True G (2003) Reiki--review of a biofield therapy history, theory, practice, and research. Altern Ther Health Med 9: 62-72.[Crossref]

36. van Sell SL (1996) Reiki: an ancient touch therapy. $R N$ 59: 57-59.[Crossref]

37. Bullock M (1997) Reiki: a complementary therapy for life. Am J Hosp Palliat Care 14: 31-33. [Crossref]
38. LaTorre MA (2005) The use of Reiki in psychotherapy. Perspect Psychiatr Care 41: 184-187. [Crossref]

39. Rand WL (2014) Reiki in Hospitals. Available at http://www.centerforreikiresearch. org/Articles_ReikiInHosp.aspx.

40. The International Association of Reiki Professionals (IARP). Available at www.iarp.org.

41. Benor DJ (2001) Spiritual Healing: Scientific Validation of a Healing Revolution (Healing Research Vol I). Southfield, MI: Vision Publications.

Copyright: $(02015$ Singg S. This is an open-access article distributed under the terms of the Creative Commons Attribution License, which permits unrestricted use, distribution, and reproduction in any medium, provided the original author and source are credited. 